\title{
Nuevos procedimientos en anestesia local en odontología: el sistema Injex ${ }^{\circledast}$
}

\author{
Fernández-Canedo C*, Machuca G**
}

\section{RESUMEN}

Una de las circunstancias que han contribuido al desarrollo de la odontología en los últimos tiempos ha sido la mejora en las técnicas anestésicas.

Sin embargo, el uso de la aguja en la mayor parte de los sistemas de anestesia dental, a pesar de ser un sistema casi indoloro, constituye un problema por la gran cantidad de pacientes que presentan fobias a la misma. Este hecho empeora cuando las poblaciones que han de ser tratadas son niños o pacientes especiales.

En esta revisión bibliográfica se exponen las ventajas e inconvenientes de las técnicas anestésicas sin aguja, y se describe un nuevo sistema aparecido en el mercado español, el sistema Injex ${ }^{\$}$.

Palabras Clave: Anestesia dental, Odontología.

\section{ABSTRACT}

In the recent years, the improvement of anesthesics methods have been one of the circunstances who had contributed to the development of Odontology.

In the other hand, sometimes is an important problem the employment of the needle in the most of anethesisc systems, because many patients present fobic situations when the needle is employed. This situation is worst when children or handicapped patients have to be treated.

In this review, the advantages and mistakes of dental anesthesia without needle is exposed, and a new method developed, called Injex ${ }^{\circledR}$, is presented.

Key words: Dental anesthesia, Odontology.

Aceptado para publicación: Enero 2004.

* Colaboradora Honoraria de Clínica Odontológica Integrada de Pacientes Espaciales. Facultad de Odontología de Sevilla.

** Profesor Titular. Clínica Odontológica Integrada de Pacientes Especiales. Facultad de Odontología de Sevilla.

Fernández-Canedo C, Machuca G. Nuevos procedimientos en anestesia local en odontología: el Sistema Injex ${ }^{\circledR}$. Av. Odontoestomatol 2004; 20-3: 131-138.

\section{INTRODUCCIÓN}

La cavidad oral se presta fácilmente a las técnicas de infiltración y bloqueo regional y es por esto que los anestésicos locales suponen la base del tratamiento del dolor en la mayoría de los procedimientos intraorales. La eficacia, simplicidad y seguridad de las técnicas de anestesia local las convierten en una opción atractiva para controlar el dolor durante los procedimientos rutinarios en la práctica odontológica (1).

Desde que los indios de Sudamérica descubrieran que masticar hojas de coca producía un efecto 
psicotrópico y conocían el efecto entumecedor que éstas producían en boca y lengua, hasta nuestros días, ha habido una larga evolución en los anestésicos y técnicas de administración de la anestesia (2).

De este modo, desde los primeros inicios de tratramientos odontológicos bajo anestesia con Morton, Wells y Rigs, ha habido una gran evolución en técnicas y en sistemas anestésicos, existiendo un gran desarrollo de jeringas y agujas, con diseños de Rynd (1845), Parvas (1852), Wood (1885) y Cook (3), hasta el actual de jeringas y carpules e incluso existiendo ya sistemas de aplicación de anestésicos sin aguja.

Toda esta evolución ha tenido como objetivo el alcanzar una técnica anestésica depurada y unos anestésicos eficaces.

\section{TIPOS DE ANESTÉSICOS}

\section{Clasificación}

Aunque hay muchos fármacos que bloquean los canales de sodio sensibles a voltaje e inhiben la generación del potencial de acción, los únicos fármacos de esta categoría clínicamente útiles son los anestésicos locales, varios fármacos anticonvulsionantes y antiarrítmicos de clase I (4).

Se utilizan principalmente con la finalidad de suprimir o bloquear los impulsos nociceptivos, sea en los receptores sensitivos, a lo largo de un nervio o tronco nervioso o en los ganglios, y tanto si la aferencia sensorial discurre por nervios aferentes somáticos o vegetativos (5).

Las moléculas anestésicas locales están formadas por una parte aromática ( que es un grupo lipofílico), unida por un enlace tipo éster o una amida a un grupo amino terciario o secundario hidrofílico el cual es una cadena lateral básica, y por lo general con sustancias sintéticas, exceptuando la cocaína $(3,4)$.

Son clasificados por ese grupo amida o éster (6). De la presencia del éster o amida en los anestésicos locales depende su susceptibilidad a la hidrólisis metabólica. Los compuestos que contienen ésteres suelen activarse en el plasma y en los tejidos por esterasas no específicas. Las amidas son más estables, y estos anestésicos tienen, en general, una semivida plasmática más larga (4). Además, tienen menos incidencia de hipersensibilidad y mayor eficacia siendo éstas las razones por las que los anestésicos locales de naturaleza amídica casi han reemplazado a los ésteres en odontología (1).

Dentro de los anestésicos que pertenecen al grupo de la amidas encontramos la articaína, bupivacaína, lidocaína, mepivacaína, prilocaína; la procaína es el prototipo de los anestésicos locales del grupo éster (6).

\section{Farmacocinética}

Los anestésicos locales varían enormemente en su rapidez de penetración en los tejidos, lo que afecta a la velocidad de bloqueo nervioso cuando se inyectan y a la rapidez de inicio y recuperación de la anestesia. También afecta a su utilidad como anestésicos superficiales para la aplicación en las membranas mucosas.

La duración de la acción es muy variable para cada anestésico; depende de la concentración y la cantidad empleada, del tipo de bloqueo seleccionado, de la existencia o no de un agente vasoconstrictor asociado, de las propiedades vasodilatadoras del propio agente y del flujo sanguíneo local. (5). El pH influye de manera notable en la actividad anestésica local, lo cual puede ser clínicamente importante. La acción anestésica local aumenta a $\mathrm{pH}$ alcalino y viceversa. De este modo, en los tejidos inflamados suele haber un ambiente ácidos y, por lo tanto, presentar resistencia a éstos fármacos (4).

\section{Mecanismo de acción}

La anestesia local consiste en la interrupción de la transmisión en un punto concreto del trayecto nervioso e incluso en la eliminación de la recepción de un estímulo. Esto se consigue actuando sobre los receptores o sobre el recorrido del nervio. Se dice que la zona a tratar está anestesiada cuando no existe la transmisión de las sensaciones desde la fuente 
de estímulo, como puede ser diente o periodonto, al cerebro (6).

Utilizados en concentraciones adecuadas inhiben de forma reversible la conducción nerviosa cuando se aplican a zonas concretas del organismo. Actúan bloqueando el inicio de la despolarización y la propagación del cambio del potencial de membrana (3), impidiendo el aumento de la conductancia al sodio dependiente de voltaje (4). Deprimen la propagación de los potenciales de acción en las fibras nerviosas porque bloquean la entrada de sodio a través de la membrana en respuesta a la despolarización nerviosa (5).

Se diferencian de la mayoría de los fármacos empleados terapéuticamente en que estos últimos, independientemente de su vía de administración, tienen un recorrido, a concentraciones considerables, por el torrente circulatorio antes de llegar a ejercer el efecto clínico que se espera de ellos; en cambio, los anestésicos locales han de realizar su acción antes de absorberse en el torrente sanguíneo, al que deberían acceder de forma lenta y progresiva de manera que su concentración fuera la más baja posible (3).

En general, los anestésicos locales bloquean la conducción en las fibras nerviosas de pequeño diámetro más rápidamente que en las fibras grandes. Las fibras más pequeñas de los nervios periféricos son fibras C no mielinizadas, por lo tanto las fibras más sensibles a los anestésicos locales son las más delgadas -A- delta y C. Dado que los impulsos nociceptivos se transportan por fibras Ad y C, la sensación de dolor se bloquea más rápidamente que otras modalidades sensitivas ( tacto, propiocepción, etc). Los axones motores, al ser de mayor diámetro, también son relativamente resistentes $(3,4)$. Son más sensibles a la anestesia las fibras de menor diámetro. En general existe un orden de pérdida de la sensibilidad: dolor, temperatura, tacto y propiocepción. Las fibras motoras son muy resistentes al bloqueo ( 5).

Conseguir éxito en la administración de la anestesia siempre ha sido una constante preocupación en odontología. Los rangos para valorar el éxito de la anestesia siempre van a depender del estado dental, pulpar o condiciones particulares de las que partamos y según las pruebas que realicemos para evaluar el efecto anestésico. Igualmente los datos para valorar el fracaso tras la aplicación de la anestesia los podremos agrupar dentro de si el fallo del material, del operador o del paciente (7).

Aplicado a la odontología, la anestesia de la pulpa cuesta más de alcanzar que la del periodonto, lo cual se explica porque el calibre de las fibras pulpares es mayor que el de las fibras de la membrana periodontal (3)

\section{Efectos indeseables}

La mejoría y avance en los productos y técnicas de anestesia local, es posiblemente uno de los logros más importantes dentro de la medicina en los últimos cien años. Hoy en día, los anestésicos pueden ser administrados con la más mínima irritación y posibilidad de reacciones alérgicas. Una variedad de productos proporcionan una rápida anestesia con la duración adecuada (8). Los anestésicos locales suelen ser considerados relativamente seguros, pero debido a la frecuencia de su uso, han de ser consideradas sus posibles reacciones adversas (6).

El contenido estándar de un carpule de anestesia incluye una amina o éster, un vasoconstrictor adrenérgico y un antioxidante. En determinados pacientes, cualquiera de estos componentes puede inducir reacciones adversas sistémicas dosis-dependientes (9).

Gran parte de los efectos indeseables y de la toxicidad (6) de los anestésicos locales depende de si hay o no absorción sistémica del fármaco, y ésta depende de: a) la dosis, b) el lugar de la inyección, particularmente en relación con la perfusión local, c) la inyección intravascular accidental, d) la rapidez de la inyección, e) la adición de vasoconstrictores, y f) las propiedades fisicoquímicas del anestésico, como liposolubilidad y fijación a proteínas tisulares (5).

Los principales efectos indeseables de los anestésicos locales afectan los sistemas nervioso central y cardiovascular, y constituyen la principal fuente de riesgo cuando se usan clínicamente. Pueden producir ansiedad $(6,10)$ manifestándose más frecuentemente como síncope, reacciones alérgicas $(4,5,6)$, metahemoglobinemia $(3,6)$, hipertermia maligna (3), parestesias en lengua o labio o anestesia prolongada 
(6), interacciones medicamentosas (3). Los efectos cardiovasculares de los anestésicos locales se deben, principalmente, a depresión miocárdica y a la vasodilatación (4). Pueden actuar sobre el corazón, los vasos y sus propios reguladores nerviosos (3).

Desde el punto de vista de la Odontología, prácticamente sólo se constatará el efecto local sobre los vasos de nuestro territorio que será de ligera vasodilatación para la mayor parte de ellos. De todos modos, cuando la concentración plasmática del anestésico local aumenta significativamente, se observa sobre el miocardio un efecto depresivo ya que desminuye su excitabilidad eléctrica, su conductibilidad y también su contractibilidad (3).

La embarazada en más susceptible a la acción cardiotóxica de estos fármacos (5).

\section{SISTEMAS DE ADMINISTRACIÓN}

\section{1) Anestesia por punción}

Consiste en la administración de la solución anestésica utilizando para su aplicación jeringa y aguja, depositando el anestésico de manera local en la zona que queramos anestesiar. En odontología, lo más común es aplicar el anestésico de manera infiltrati$v a$, inyectándolo alrededor de las terminaciones nerviosas o de fibras nerviosas que no son macroscópicamente identificables o mediante bloqueo nervioso, cuando la inyección del anestésico local se hace lejos de las terminaciones nerviosas, sea en un tronco nervioso importante o en un ganglio nervioso y lógicamente el efecto anestésico es muy superior a las técnicas infiltrativas. A lo largo de los años se ha producido una evolución muy evidente en el diseño de las jeringas, desde las primeras que fueron diseñadas por Rynd, Parvas o Wood hasta las actuales, en las que se conserva el sistema básico de carpule, jeringa y aguja (3)

\section{2) Anestesia sin aguja (inyectores a presión)}

Los inyectores a presión no pueden considerarse como una novedad ya que su uso odontológico comenzó el año 1958 con Magretis y cols., pero actualmente se intenta su reintroducción.

Este sistema de aplicación de anestesia se basa en que el líquido, pasando a gran presión y velocidad por los pequeños orificios de la punta del inyector, depositándose en el tejido subcutáneo, siendo capaz de atravesar membranas como la epidermis y la mucosa bucal. Permiten conseguir la penetración de 0.2- $0.4 \mathrm{ml}$ de la solución anestésica en áreas que van desde los $5 \mathrm{~mm}$ a $1 \mathrm{~cm}$ de diámetro. La entrada de líquido es indolora pero se debe ir con cuidado de que el paciente no se mueva ya que entonces se pueden traumatizar innecesariamente los tejidos blandos (7).

Aunque este sistema fue diseñado para tratamiento dental, su versatilidad y simplicidad hace que se pueda usar además para otras aplicaciones médicas donde se necesite un campo anestesiado relativamente pequeño. Especialmente está indicado para odontología pediátrica y para pacientes con fobia a las agujas. Proporciona una zona anestesiada amplia para la colocación de agujas hipodérmicas o como paso previo a la anestesia definitiva, para procedimientos como biopsias, aspiraciones medulares así como pequeñas intervenciones (11).

Este tipo de anestesia presenta una alta aceptación por parte de los adultos, en un $90 \%$, y de los niños, un $75 \%$ (7) y en varios estudios se ha usado el sistema de anestesia sin aguja para la premedicación antes de la inducción de la anestesia, como la administración de midazolam, tiene muchas ventajas incluyendo una reducción de la ansiedad asociada al miedo a las agujas y a las heridas provocadas por el sistema anestésico convencional $(12,13,14,15)$.

En un estudio realizado sobre niños, se ha observado que tras la administración de premedicación con el sistema sin aguja ha sido efectiva, rápida y segura y en cinco minutos ha sido posible localizar una vena (12). Además se ha observado la eficacia en la administración de lidocaína con el sistema sin aguja para la cateterización intravenosa (16). A este respecto, Cooper y cols., recomiendan que para anestesiar la zona previa a canulación venosa con el sistema sin aguja es preferible que se haga en áreas donde el espacio subcutáneo es más profundo (17). 
El uso de estos sistemas sin aguja, no reportan prácticamente ningún riesgo, y su uso es sencillo, no obstante, se deben tener ciertas precauciones con respecto a la posible contaminación de paciente a paciente. En este sentido, Weintraub, tras realizar un estudio sobre los capuchones del sistema de anestesia sin aguja, aisló la presencia de Streptoccocus crista, bacteria comúnmente aislada en la saliva. Eso hace que exista una contaminación de todas las superficies donde se use el mismo capuchón. Recomienda cambiar el capuchón en cada uso, aunque eso sólo no es suficiente para evitar la contaminación. Hace falta poner la cabeza del inyector al $2 \%$ de glutaraldehído posteriormente enjuagado con agua estéril y el cambio del capuchón usado por uno estéril entre usos ha demostrado cortar el crecimiento bacteriano y prevenir los casos de contaminación cruzada (18).

El miedo a las inyecciones en los procedimientos dentales es común entre nuestros pacientes. En las últimas décadas, se está investigando sobre nuevos sistemas y técnicas de aplicación de anestésicos donde se reduzca el miedo a la inyección y se mejore la anestesia local (19).

Muchos pacientes tienen miedo al pinchazo en la anestesia local. De hecho, la mayoría de ellos lo citan como primera razón a la hora de evitar la visita al dentista (20). En odontología, el manejo del dolor es un componente crítico en el cuidado del paciente y cuando no se consigue una correcta anestesia, el resultado del estrés del paciente y el dentista, puede ser significativo (21).

El sistema de anestesia sin aguja aparece como una alternativa eficaz para la aplicación de anestesia tópica en la mucosa oral. No obstante, su uso para anestesia pulpar es cuestionable. Las principales ventajas de este sistema son la eliminación de el daño que puede ocasionar el pinchazo y una excelente aceptación por parte del paciente (7).

\section{EL SISTEMA INJEX ${ }^{\circledast}$ DE ANESTESIA DENTAL SIN AGUJA}

Los inyectores a presión están adaptados para su uso en la consulta dental. El sistema consta de:

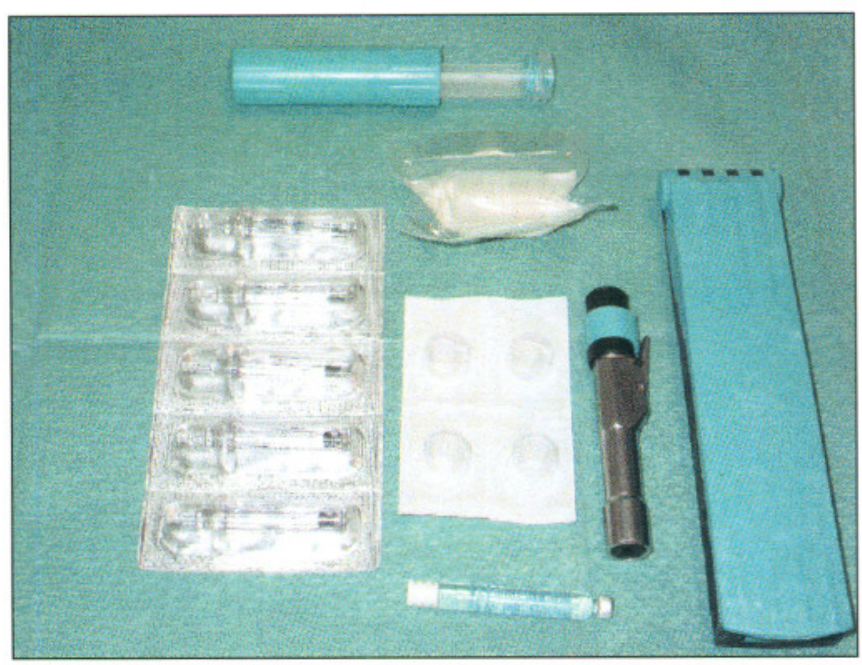

Fig. 1. Componentes de los que consta el sistema Injex.

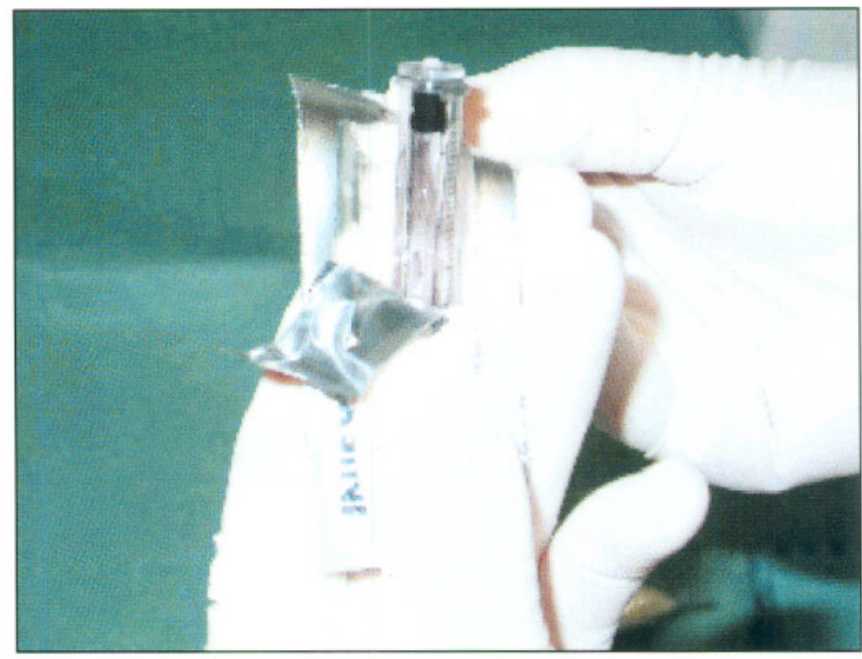

Fig. 2. Extracción de la ampolla Injex del blister.

Ampolla esterilizada de un solo uso, la cual está diseñada para ser llenada por el líquido anestésico, mediante un transportador y un adaptador para el transportador, desde el carpule de anestesia convencional a la ampolla. La ampolla tiene una espiga móvil, que impulsa el anestésico durante el proceso de la inyección a gran velocidad a través de una microapertura en la punta de la ampolla, con lo que se produce, con alta presión, la salida de líquido que atraviesa la piel. A la ampolla se le adapta el Silitop, cuyo uso solamente es en combinación con la ampolla Injex. Sirve para no traumatizar la zona donde apoya la jeringa Injex. La presión del líquido anestésico la confiere el inyector, que se activa con la Reset box. El inyector se introduce en la Reset box y se activa. De este modo está preparado para la 


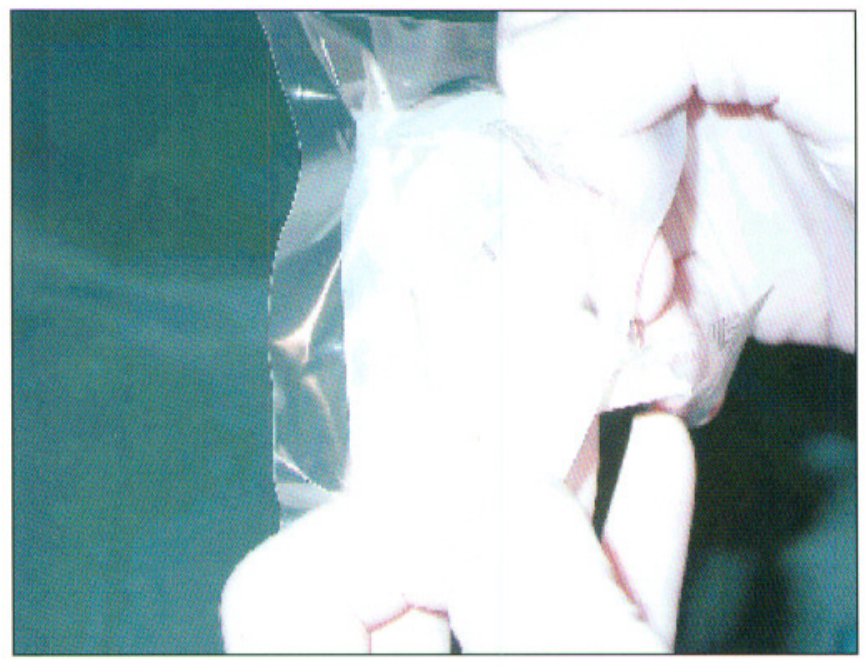

Fig. 3. El adaptador para el transportador en su recipiente estéril.

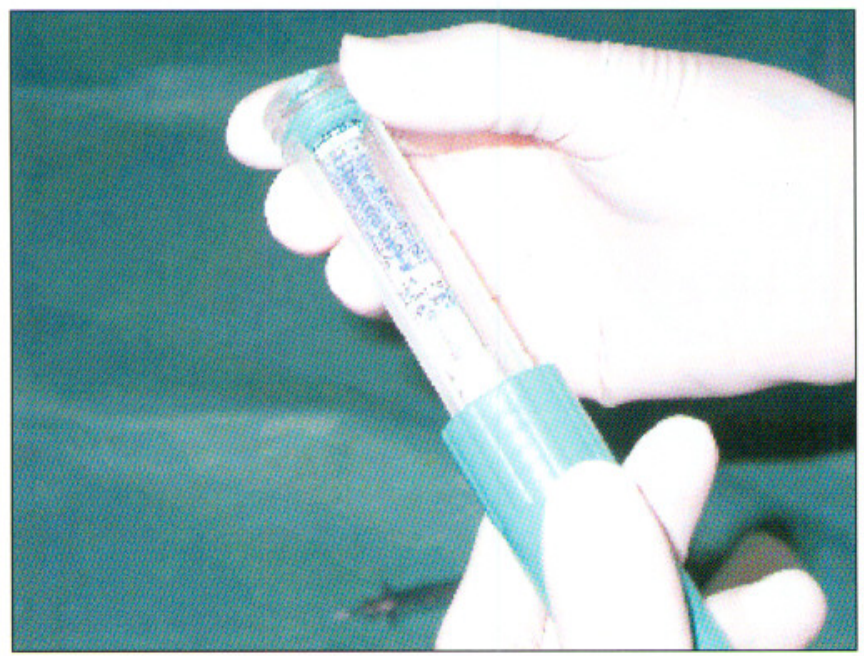

Fig. 4. Introducción y adaptación del carpule standar en el transportador.

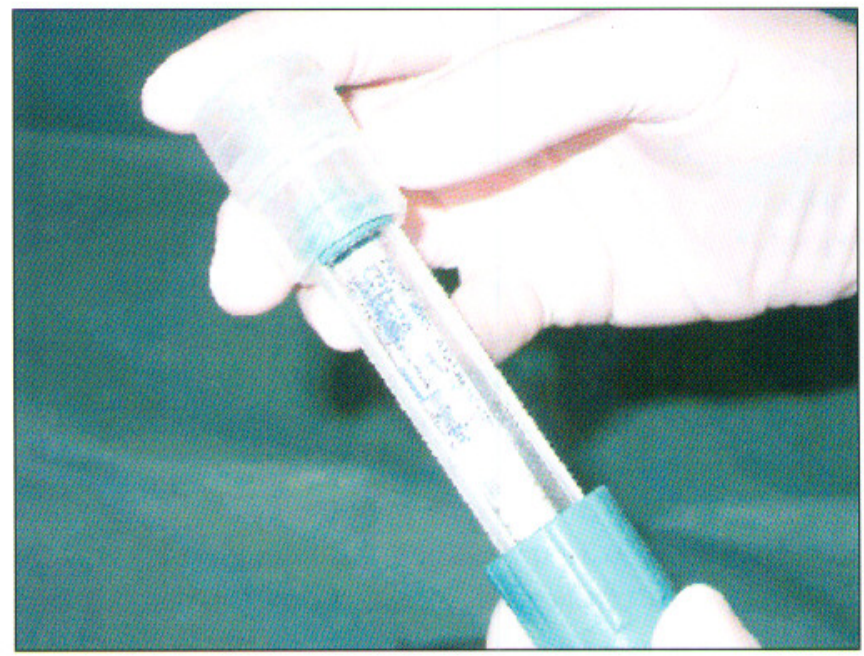

Fig. 5. Enroscado del adaptador.

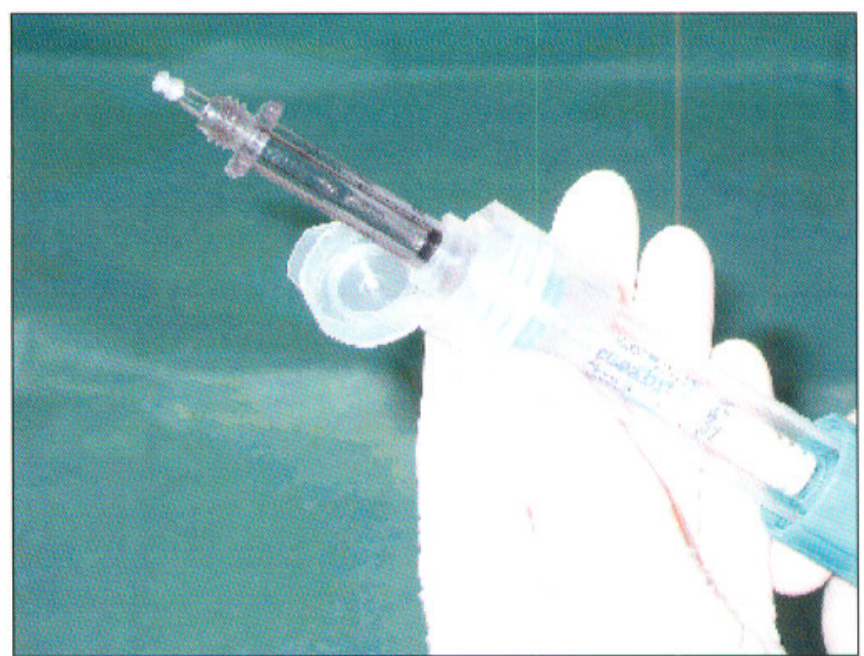

Fig. 6. Adaptación del carpule Injex y llenado del mismo de anestésico desde el carpule estándar.
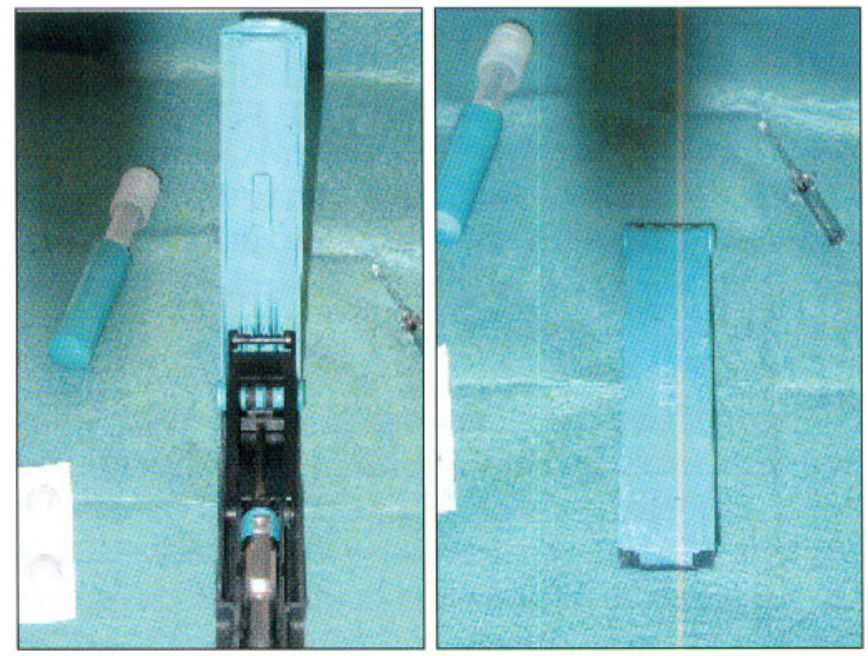

Fig. 7. Reset box o caja activadora. Cerrándola se activa el injector que confiere la fuerza de injección.

colocación de la ampolla previamente cargada, y posteriormente para la administración del anestésico. Una vez descargada la ampolla, el inyector necesita ser activado nuevamente (Figs. 1-8).

Está diseñado este sistema para la administración submucosa del anestésico en la zona anterior, tanto en la zona superior como inferior, siendo el lugar de inyección entre los ápices de los dientes y en la zona más apical dentro de la encía adherida. La ampolla ha de estar perpendicular en la zona de inyección, manteniéndola en esta posición unos segundos una vez efectuada la aplicación del anestésico, evitando de este modo el leve sangrado posterior (Fig 9) . 


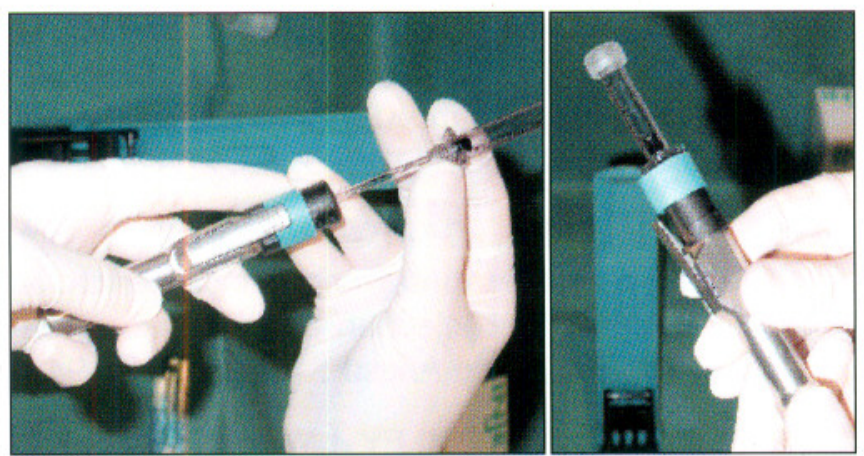

Fig. 8. Enroscado del carpule Injex en el injector activado. Desbloqueo del sistema de seguridad, y colocación del capuchón de goma protector al carpule Injex. La jeringa está lista para su uso.

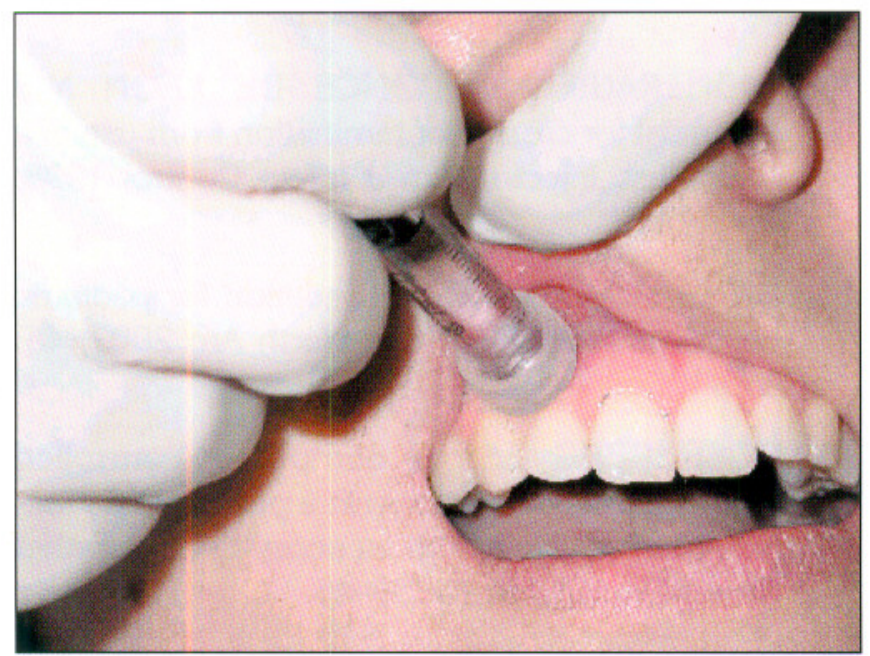

Fig. 9. Posición ideal para la colocación del carpule Injex en la anestesia de zonas anterosuperiores.

Saravia y Bush (11), en un estudio sobre 34 niños con edades comprendidas entre 5 y 15 años, a los que se les realizó tratamientos dentales completos usando el sistema Syrijet demostraron que era estadísticamente significativo un clara diferencia de estos chicos a favor de la aplicación de la anestesia sin aguja (25 de ellos lo preferían). De igual modo, Munshi y cols., en un estudio sobre 100 niños con edades de entre 3 y 13 años, observaron que el uso de la anestesia convencional en clínica daba un mal comportamiento en los niños, no siendo así con el uso del sistema sin aguja (11).

Su uso puede estar indicado para la colocación de hilos retractores, incisiones para drenaje de abcesos, colocar bandas o mantenedores de espacio. Así mismo, da buen resultado en la encía gingival libre, no así en la anestesia pulpar de los incisivos laterales permanentes (7).

Presenta raros efectos secundarios. Cuando no, puede aparecer un hematoma tras la aplicación de la anestesia (7).

\section{VENTAJAS E INCONVENIENTES DEL SISTEMA DE ANESTESIA SIN AGUJA}

\section{Ventajas:}

- rápido efecto anestésico

- predecible efecto anestésico local en los tejidos blandos

- eliminación del pinchazo

- bajo riesgo de inyección intravascular

- alta aceptación por parte del paciente

- mejora del comportamiento de los niños en la consulta a la hora de aplicar este tipo de anestesia local (7).

\section{Inconvenientes:}

- elevado coste

- miedo por parte del paciente tras notar la presión y escuchar el ruido al administrar la anestesia

- posibilidad de aparición de hematomas

- eficacia cuestionable en la anestesia pulpar (7)

- posibilidad de contaminación cruzada, tras el contacto directo con superficies contaminadas, si no se toman las medidas de prevención adecuadas (18).

\section{BIBLIOGRAFÍA}

1. HIGGINS: Anestesia en odontología: Anestesia en cirugía periodontal. En Clin Od de Nort, McGraw- Hill Interamericana, vol 2: 296-319, 1999.

2. YAGIELA J: Anestesia tipo Office- Based en odontología. Pasado, presente y tendencias futuras. En Clin Od de Nort, McGraw- Hill Interamericana, vol 2 : 223- 39, 1999.

3. AYTÉS BL, ESCODA CG. Anestesia odontológica. Eds. Avances. Madrid, 1997. 
4. RANG HP, DALE MM, RITTER JM: Anestésicos locales y otros fármacos que afectan a los canales iónicos. EN Rang HP, Dale MM, Ritter JM. Farmacología. Marcourt. 2000.

5. HURLÉ MA: Anestésicos locales. En FLÓREZ J, ARMIJO JA, MEDIAVILLA A. Farmacología humana. Masson. 2002.

6. HAAS DA: An update on local anesthetics in dentistry. J Can Dent Assoc 2002; 68: 546-51.

7. WONG JK: Adjunts to local anesthesia: separating fact from fiction. J Can Dent Assoc 2001; 67: 391-7.

8. HAWKINS JM, MOORE PA: Local anesthesia: advances in agents and techniques. Dent Clin North Am 2002 ; 46: 719-32.

9. FINDER RL, MOORE PA: Adverse drug reactions to local anesthesia. Dent Clin North Am 2002 ; 46: 747- 57.

10. KAUFMAN E, GOHARIAN S, KATZ Y: Adverse reactions triggered by dental local anesthetics: a clinical survey. Anesth Prog 2000; 47: 134-8.

11. SARAVIA ME, BUSH JP: The needleless syringe: efficacy of anesthesia and patient preference in child dental patients. J Clin Pediatr Dent 1991; 15: 109-12.

12. BENNET $J$, NICHOLS $F$, ROSENBLUM $M$, CONDRY J: Subcutaneous administration of midazolam: a comparison of the Biojet jet injector with the conventional syringe and needle. $\mathrm{J}$ Oral Maxillofac Surg 1998; 56: 1249- 54.

13. GELDNER G, HUBMANN M, KNOLL R, JACOBI K: Comparison between three transmucosal routes of administration of midazolam in children. Paediatr Anaesth 1997; 7: 103-9.

14. DOMINO EF, ZSIGMOID EK, KOVACS V, OLAJOS B, FEKETE G: A new route, jet injection for anesthetic induction in children. IV. Midazolam plasma levels. Int J Clin Pharmacol Ther 1998; 36: 458-62.
15. GREENBERG RS, MAXWELL LG, ZAHURAK M, YASTER M: Preanesthetic medication of children with midazolam using the Biojector jet injector. Anesthesiology 1995; 83: 264-9.

16. ZSIGMOND EK, DARBY P, KOENING HM, GOLL EF: Painless intravenous catheterización by intradermal jet injection of lidocaine: a randomised trial. J Clin Anesth. 1999; 11: 87-94.

17. COOPER JA, BROMLEY LM, BARANOWSKY AP, BARKER SG: Evaluation of a needle- free injection system for local anaesthesia prior to venous canulation. Anaesthesia 2000; 55: $247-$ 50.

18. WEINTRAUB AM, PONCE DE LEÓN MP: Potencial for cross- contamination from use of a needleless injector. Am J Infect Control 1998; 26: 442-5.

19. WEBB MD, MOORE PA: Sedation for pediatric dental patients. Dent Clin North Am 2002; 46: 803-14.

20. ROSENBERG ES. A computer- controlled anesthetic delivery system in a periodontal practice: patient satisfaction an acceptance. J Esthet Restor Dent 2002; 14: 39-46.

21. FRIEDMAN MJ: New advances in local anesthesia. Compend Contin Educ Dent 2000; 21: 432 6, 438, 440 .

22. MUNSHI AR, HEDGE A, BASHIR N: Clinical evaluation of the efficacy of anesthesia and patient preference using the needle- less jet syringe in pediatric dental practice. $\mathrm{J}$ Clin Pediatr Dent 2001, Winter; 25: 131- 6.

\section{CORRESPONDENCIA}

Guillermo Machuca Portillo.

Facultad de Odontología. Universidad de Sevilla. c/ Avicena s/n, 41009-Sevilla. Teléfono 954481128. gmachuca@us.es 